OPEN ACCESS

Edited by:

Martin James Turner,

Staffordshire University,

United Kingdom

Reviewed by:

Chris Englert,

University of Bern, Switzerland Henrik Gustafsson,

Karlstad University, Sweden

Gianluca Serafini,

San Martino Hospital (IRCCS), Italy

*Correspondence:

Marie Ottilie Frenkel

marie.frenke/@issw.uni-heidelberg.de

Specialty section:

This article was submitted to

Movement Science and Sport

Psychology,

a section of the journal

Frontiers in Psychology

Received: 28 February 2019

Accepted: 10 July 2019

Published: 08 August 2019

Citation:

Frenkel MO, Brokelmann J,

Nieuwenhuys A, Heck R-B, Kasperk $C$, Stoffel $M$ and Plessner $H$ (2019) Mindful Sensation Seeking: An

Examination of the Protective Influence of Selected Personality

Traits on Risk Sport-Specific Stress.

Front. Psychol. 10:1719.

doi: 10.3389/fpsyg.2019.01719

\section{Mindful Sensation Seeking: An Examination of the Protective Influence of Selected Personality Traits on Risk Sport-Specific Stress}

\author{
Marie Ottilie Frenkel1*, Joana Brokelmann², Arne Nieuwenhuyss ${ }^{3,4}$, Robin-Bastian Heck¹, \\ Christian Kasperk ${ }^{5}$, Martin Stoffel ${ }^{6}$ and Henning Plessner ${ }^{1}$
}

${ }^{1}$ Institute of Sports and Sports Sciences, Heidelberg University, Heidelberg, Germany, ${ }^{2}$ Psychological Institute, Goethe University Frankfurt, Frankfurt, Germany, ${ }^{3}$ Department of Exercise Sciences, The University of Auckland, Auckland,

New Zealand, ${ }^{4}$ Behavioural Science Institute, Radboud University, Nijmegen, Netherlands, ${ }^{5}$ Department of Internal Medicine I and Clinical Chemistry, Steroid Laboratory, University Hospital Heidelberg, Heidelberg, Germany, ${ }^{6}$ Institute of Medical

Psychology, University Hospital Heidelberg, Heidelberg, Germany

Stress often has a negative influence on sports performance. Stress-induced decreases in performance can be especially disastrous for risk sports athletes, who often put their life at risk when practicing their sport. Therefore, it is of great importance to identify protective factors in stressful situations in risk sports. On average, risk sports athletes score extremely high on the personality trait sensation seeking. At the same time, theoretical considerations about dispositional mindfulness suggest that mindful athletes can handle stress more effectively. The main goal of this experiment is to examine the influence of sensation seeking and mindfulness on the stress response to a risk sport-specific stressor. To induce stress, 88 male students completed the Heidelberg Risk Sport-Specific Stress Test (HRSST) which utilizes fear of falling as the stressful event during a climbing exercise. Psychological (anxiety) and physiological (cortisol) responses were measured at multiple time points before and after the HRSST to determine the severity of the stress response. In reaction to the stressor, a significant increase in self-reported state anxiety, but no significant increase in cortisol were observed. The mindfulness subscale external observation correlated positively with anxiety in the climbing wall, sensation seeking and the anxiety scales after the jump correlated negatively and sensation seeking predicted anxiety subscales after the jump in hierarchical regression analyses. However, mindfulness did not predict anxiety measures. Neither sensation seeking nor mindfulness correlated significantly with cortisol levels. The results suggest that high sensation seekers perceive a risk sport-specific stressor as less stressful. The missing physiological response might be explained by the Cross-Stressor-Adaptation-Hypothesis and particularities of the sample. Good internal observers might be especially aware of their need of stimulation and new experiences, which in turn might explain the higher experience-seeking scores. 
Future studies should further examine the role of mindfulness in stressful situations and the interaction of its subscales with sensation seeking. The current experiment offers new possibilities for adjoining research fields at the interface between sports sciences, psychology and medicine: The findings can be transferred to high risk professions such as police officers, firefighters and military forces (e.g., for selection processes or for interventions).

\section{Keywords: mindfulness, anxiety, cortisol, sensation seeking, risk sport-specific stress}

\section{INTRODUCTION}

Athletes plunge from mountains only wearing a wingsuit, free climbers scale high rock faces without any form of protection, and surfers aim to ride huge waves before they break the shores. High risk sports athletes who practice those demanding activities frequently set their physical integrity at risk, making it crucial to deliver peak-performance. Typically, highly demanding situations induce distress and therefore threaten peak performance-delivery (Paulus et al., 2009; Röthlin et al., 2016). In contrast, however, some risk sports athletes are known to report positive rather than negative responses and emotions during task execution (Arijs et al., 2017; Frenkel et al., 2018b; Houge Mackenzie and Brymer, 2018), often resulting in peak performance. Why do some people report not being afraid in such extreme situations? Empirical studies point to specific personality traits that may influence stress and performance in (high risk) sports (Plessner et al., 2009; Allen et al., 2013; Röthlin et al., 2016). Pertinent to the current study, one of these traits - sensation seeking - is prevalent among risk sports athletes and seems to have stress-buffering and performancefacilitating effects (Anshel and Anderson, 2002; Ruedl et al., 2012; Frenkel et al., 2018b). In addition to being high in sensation seeking, narrative research indicates that risk sports athletes describe their strengths in risky situations in words that resemble mindful mindsets (Arijs et al., 2017; Houge Mackenzie and Brymer, 2018). Based on these data, an intriguing question is whether dispositional mindfulness may contribute to risk sports athletes' positive emotional responses and functioning in highly demanding situations. Building on existing narrative research, the aim of the present study is to provide an experimental examination of the protective influence of sensation seeking and mindfulness on risk sport-specific stress responses.

\section{Stress}

According to Lazarus and Folkman's (1984) transactional model of stress, stress results from the athlete's subjectively perceived discrepancy between the demands being placed by the environment and coping resources available in a particular situation. High risk sports athletes are often required to respond to situations which threaten their physical integrity or psychological well-being (Breivik, 1999b). In such circumstances, they usually have little opportunity to make corrective decisions, for example, deciding to interrupt a first free-solo ascent in rock climbing to try again later, or correcting errors and avoiding structures while flying in a wingsuit at a speed of over $200 \mathrm{mph}$. When an individual perceives environmental demands to outweigh their coping resources, a negative and unpleasant psychological state ensues, characterized by feelings of stress and anxiety (Lazarus, 2000). In this respect, anxiety is regarded as an aversive emotional and motivational state that arises when facing uncertainty or a perceived existential threat (Eysenck et al., 2007).

Critical incidents in high risk sports hold high levels of novelty, uncontrollability and personal threat of injury or death (Breivik, 1999b). Besides showing a psychological response (as indicated above), the human body also shows a physiological response to such situations (Campbell and Ehlert, 2012), including activation of the fast reacting sympathetic adrenomedullary system (SAM) - which triggers the release of adrenaline and noradrenaline - and the slower hypothalamicpituitary-adrenal (HPA) axis, which triggers the release of cortisol from the adrenal cortex. Indeed, several studies have shown that critical incidents place high physiological demands on athletes. For example, although physiological response patterns were slightly inconsistent across studies, it has been found that athletes showed increases in subjective stress (e.g., self-reported anxiety) and salivary cortisol in response to various (sportspecific) experimental stress protocols (Lautenbach et al., 2014; Lautenbach, 2017; Frenkel et al., 2018b).

In general, both psychological and physiological stress responses are associated with impairments in cognitive performance (Eysenck et al., 2007) as well as with a decrease in sports performance (Lautenbach et al., 2014; Frenkel et al., 2018b; e.g., see Nieuwenhuys and Oudejans, 2012, 2017, for a review).

\section{Sensation Seeking}

With regard to high risk sports, one personality trait that may protect risk sports athletes from negative effects of stress is "sensation seeking" (Zuckerman, 1994, 1996). Indeed, risk sports athletes have been shown to score extremely high on measures of sensation seeking (Breivik, 1999b; Ruedl et al., 2012) which is defined as the "seeking of varied, novel, complex, and intense sensations and experiences and the willingness to take physical, social, legal, and financial risks for the sake of such experience" (Zuckerman, 1994, p. 27).

According to the psychobiological model of sensation seeking (Zuckerman, 1994, 1996), individuals differ in their optimal levels of physiological arousal and the stimulation required to establish a certain level of arousal. In contrast to low sensation seekers (LSS) - who feel better in less stimulating environments -, high sensation seekers (HSS) tend to have lower baseline levels of dopamine and norepinephrine, which leads these individuals to continuously seek new and intense sensations to maintain their optimal levels of arousal (Zuckerman, 2007). 
In the context of the transactional model of stress (Lazarus and Folkman, 1984), HSS may be hypothesized to differ from LSS with respect to (a) their primary appraisal of the performance environment (e.g., lower perceived demands); (b) their perceived ability to cope (e.g., more resources) and, hence, may be expected to (c) show reduced psychological responses (e.g., lower levels of anxiety), as well as (d) reduced physiological responses (e.g., lower levels of salivary cortisol).

Indeed, with regards to (a), Franken et al. (1992) showed that HSS in comparison to LSS tended to judge risky and dangerous situations as less threatening and therefore postulate negative outcomes as less likely to occur. With regard to (b), empirical studies in sports are more scarce. Nevertheless, one study with high school athletes confirmed sensation seeking as a stress-resiliency factor, with HSS reporting better stress management coping skills than LSS (Smith et al., 1992). Regarding psychological responses (c), little is known about the relationship between sensation seeking and anxiety. Examining a sample of university students, Franken et al. (1992) found sensation seeking to be negatively correlated with anxiety. However, in a sample of parachute jumpers, only one out of four subscales of sensation seeking correlated negatively with state anxiety (Breivik et al., 1998) and - more recently - in a sample of 30 sports students, HSS did not show significantly lower anxiety than LSS in response to a high risk sport-specific stressor (Frenkel et al., 2018b). Finally, regarding physiological responses (d), high sensation seeking has been found to be related to lower baseline levels of cortisol (Shabani et al., 2011) and an attenuated cortisol response to stress (Couture et al., 2008), indicating that HSS might tolerate new, potentially stressful experiences better than LSS. Breivik (1999a) did not find a significant correlation between cortisol and sensation seeking in a sample of extreme sports athletes. However, in a recent experimental study, Frenkel et al. (2018b) confirmed that HSS showed lower levels of cortisol in response to a risk sport-specific stressor than LSS. Taken together, these data ("a," "b," "c," and "d") indicate that HSS appraise demanding performance environments more positively and exhibit psychological and physiological responses that allow them to perform better in stressful, high risk sports situations than LSS.

\section{Mindfulness}

In contrast to being reckless, risk sports athletes describe the use of internal strategies in risky situations that appear to reflect mindful mindsets (Arijs et al., 2017; Houge Mackenzie and Brymer, 2018): Including high present-moment awareness, high attunement with the environment, a simultaneous internal focus, as well as the use of deliberate value-guided action. Through well-tuned knowledge of their own physical and psychological capacities and limitations, risk sports athletes' actions are often guided by a "leave your ego at the door" mentality (Arijs et al., 2017, p. 7). Hence, in absence of (high levels of) anxiety - which often serves as a natural "brake" on behavior in LSS - HSS" mindful mindsets may allow them to make required corrective decisions when conditions are unsafe or suddenly turn aversive (such as the wind in the wrong direction for BASE jumpers).
Mindfulness is considered a specific kind of attention direction (Kabat-Zinn, 1994). Following an operational definition given by Bishop et al. (2004), mindfulness can be divided in two components: The process of continuous direction of attention and the inner attitude with which this process is carried out (openness, acceptance, self-support). A central feature of mindfulness is "centering" in the presence. Mindfulness directly impacts human behavior by interrupting automatized reaction patterns and by replacing them with flexible actions appropriate for the respective situation (Kabat-Zinn, 1994).

Different competing approaches attempt to explain positive mechanisms of trait mindfulness on well-being and health (Shapiro et al., 2006; Brown et al., 2007; Creswell and Lindsay, 2014; Creswell et al., 2019). Based on this work, and in context of the transactional model of stress (Lazarus and Folkman, 1984), highly mindful in contrast to lowly mindful individuals may be hypothesized to (a) show more favorable appraisals of their performance environment, (b) evaluate their coping resources more positively, and - hence - exhibit (c) reduced psychological responses (e.g., lower levels of anxiety), and (d) reduced physiological responses (e.g., lower levels of salivary cortisol).

Indeed, with regards to (a), the mindfulness stress buffering account (Creswell and Lindsay, 2014; Creswell et al., 2019) states that trait mindfulness mitigates stress assessment because stressors are observed with acceptance and equanimity which, in turn, buffers primary threat appraisals. In line with this assumption, Brown et al. (2012a) demonstrated that mindfulness may buffer attentional reactivity to threatening stimuli. Regarding (b), through the buffering of primary threat appraisals, mindfulness should facilitate positive secondary appraisals in favor of coping resources, decrease subsequent rumination, and increase effective coping strategies (Creswell and Lindsay, 2014). Mindful persons are found to possess better emotion-regulation abilities: Negative emotions are avoided less often (Shapiro et al., 2006). Moreover, as negative states are avoided only to a minor extent, a stronger voluntary exposition to negative emotions like anxiety takes place. As a result, a desensitization concerning anxiety responses occurs (Brown et al., 2007; Shapiro et al., 2006). In the context of sports, Josefsson et al. (2017) showed indirect effects of dispositional mindfulness on coping via rumination and emotion regulation. In line with these effects, regarding (c), research confirms that mindfulness is associated with reduced (i.e., less negative) psychological responses to stress. Outside the context of sports, research using different approaches (correlational, quasi-experimental, and laboratory studies) has shown that trait mindfulness is related to decreased levels of trait and state anxiety (e.g., Brown and Ryan, 2003; Arch and Craske, 2010). Within the sports context, Röthlin et al. (2016) showed that in elite athletes, trait mindfulness is negatively related to cognitive competitive trait anxiety, thereby helping them to perform better. Finally, regarding the physiological response to stress (d), the mindfulness stress buffering account (Creswell and Lindsay, 2014; Creswell et al., 2019) suggests that mindful individuals should exhibit increased activation of regulatory pathways in the prefrontal cortex, whilst reducing bottomup stress-reactivity (e.g., HPA axis responses), thus inhibiting 
cortisol production and release from the adrenal cortex. Recent laboratory studies with healthy participants seem to confirm these assumptions (Arch and Craske, 2010; Brown et al., 2012b; Manigault et al., 2018). However, in the context of sports, there is a lack of experimental studies investigating the link between trait mindfulness and cortisol in response to a sportspecific stressor.

\section{The Current Study}

Integrating the literature, sensation seeking and trait mindfulness are described as personality characteristics that could potentially contribute to effective stress regulation and performance in demanding (high risk) sports situations (Kabat-Zinn et al., 1985; Smith et al., 1992; Breivik, 1999a; Röthlin et al., 2016; Frenkel et al., 2018b). However, there is a shortage of experimental studies in the context of sports and, to our knowledge, there is no research investigating the role of sensation seeking AND mindfulness within one study. Against this background - and based on Lazarus and Folkman's (1984) transactional model of stress -, the present experimental study investigates how sensation seeking and mindfulness affect individuals' psychological (anxiety) and physiological (cortisol) response to a sport-specific stressor and whether mindfulness can explain additional variance in the stress response beyond sensation seeking.

- Hypothesis (H) la and b: Sensation seeking is negatively associated with (a.) state anxiety and (b.) salivary cortisol, in response to the Heidelberg Risk Sport-Specific Stress Test (HRSST).

- H2a and b: Mindfulness is negatively associated with (a.) state anxiety and (b.) salivary cortisol, in response to the HRSST.

- H3a and b: Sensation seeking explains a significant proportion of variance in (a.) state anxiety and (b.) salivary cortisol, in response to the HRSST.

- H4a and b: Beyond sensation seeking, mindfulness explains a significant proportion of variance in the prediction of (a.) state anxiety and (b.) salivary cortisol, in response to the HRSST.

\section{MATERIALS AND METHODS}

\section{Participants}

During pre-selection, a sample of $N=207$ male sports students of the Heidelberg University $(M=22.9, S D=3.3)$ were screened for eligibility based on exclusion criteria that included sports habits and health condition. Participants were excluded from the study when they had more than $5 \mathrm{~h}$ of climbing experience $(n=68$; to maximize the effectiveness of the stress induction; Ogden, 2012), or reported a particular fear of heights $(n=17)$, consumed medication containing cortisol $(n=1)$, or had injuries $(n=14)$. If none of the exclusion criteria were met, participants were invited for the experiment. Nineteen persons were not available or refused to participate.
Following the screening process, 88 male students, aged between 18 and $31(M=22.5, S D=2.8)$, were deemed eligible and agreed to participate in this study. Two persons had to be excluded from the entire analyses because one participant did not jump and the other consumed branched amino acids and creatine during the experiment.

The most frequently reported types of sports in the sample (multiple answers allowed) were soccer (21.9\%), fitness (12.5\%), and weight training (10.2\%). Concerning high risk sports, 13 persons had engaged in downhill mountain biking, three persons had done a bungee jump, three persons had engaged in different kinds of surfing activities and one person had done skydiving before. For medium risk sports, martial arts and American Football/rugby were mentioned by one participant, respectively, and skiing was reported by two participants. On average, the participants had $8.6 \mathrm{~h}$ of sports practice per week $(S D=3.7)$ and rated their fitness on average as 71.0 on a scale ranging from 1 to $100(S D=18.3)$. Participants gave written informed consent and were compensated for their participation (15 euros). The procedures were approved by the local Ethics Commission, a university board associated with the Faculty of Behavioural and Cultural Studies.

\section{Design}

This study is embedded in a bigger between-within-subjectdesign-study (Experiment 3 of Frenkel, 2018). In this study, participants were randomly assigned to an ego depletion vs. control group, while their stress parameters were assessed multiple times in the course of the investigation (see Figure 1). A state of ego depletion was induced using a 10-min copying task (Bertrams et al., 2010). Participants in the ego depletion group were instructed to copy a text about the history of the German city of Mannheim as fast and error-free as possible, while leaving out the letters " $e$ " and " $n$ ". Because these two letters appear frequently in the German language, this variation of the copying task can be considered strenuous. Participants in the control group had to copy the text without leaving out any letters. They were also instructed to copy the text as fast and error-free as possible. Effects of the ego depletion are presented elsewhere (Frenkel, 2018). The analyses were conducted with both the experimental and the control group and the authors controlled for the influence of the experimental condition (see below).

\section{Procedure}

In this study, the psychological and physiological responses to a sport-specific stressor were tested at six measurement points (for an overview see Figure 1). To adequately represent the situational demands in risk sports, we conducted the HRSST (Frenkel et al., in press), which has been introduced as an innovative, externally valid and standardized stress induction protocol. This protocol uses a climbing task with a subsequent "jump into the rope" that leads to a fall of about $3 \mathrm{~m}$ to induce stress. Participants were asked not to consume caffeine, juices, food, nicotine or alcoholic beverages within $1 \mathrm{~h}$ prior to the experiment. In accordance with the recommendations of Kudielka et al. (2009), the effect of circadian hormone rhythms was minimized by holding testing time relatively constant and conducting all 


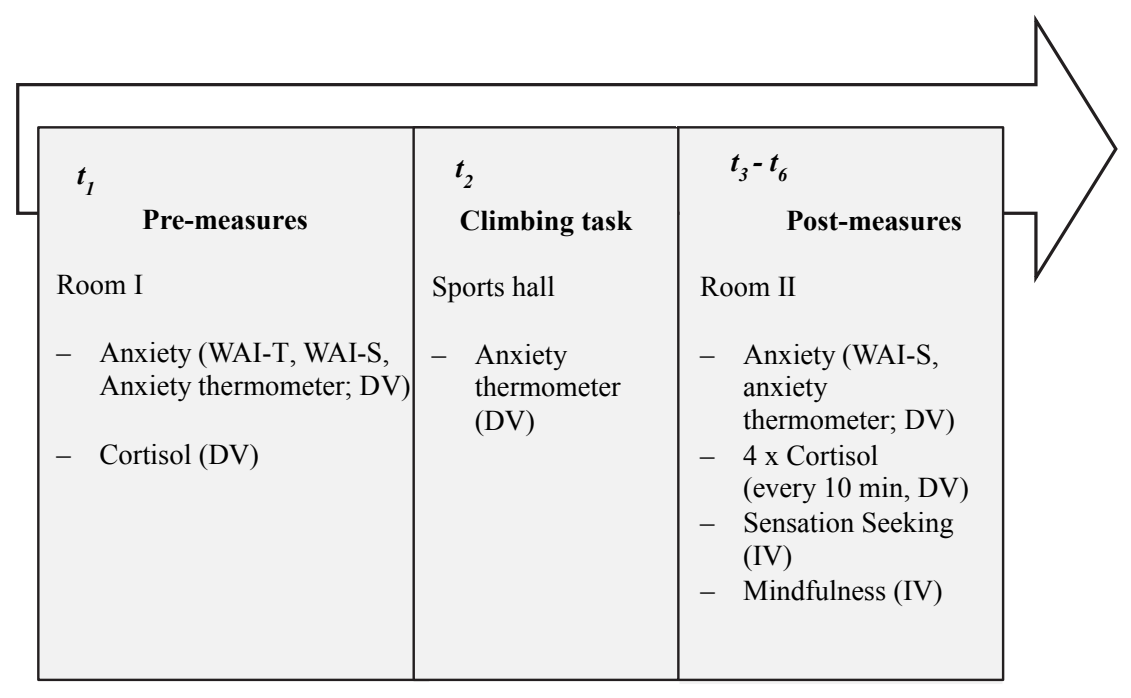

FIGURE 1 | Graphical summary of the procedure, measures, and measurement points. IV: independent variable, DV: dependent variable.

sessions in the afternoon (between 2 and 6 p.m.). Based on the idea that the stress induction and, consequently, the increase in cortisol are maximal for unpredictable tasks (Kirschbaum et al., 1993), baseline measurements before the HRSST were taken in a room outside the sports hall. This set-up ensured that participants did not develop any expectations about the upcoming climbing task. For reasons of standardization, the study followed a written protocol that described both the test procedure as well as the instructions given by the investigators.

At the first point of measurement $(t 1)$, participants filled out questionnaires for state and trait anxiety, the first salivary sample (baseline measurement) was taken and participants completed the copying task to manipulate their self-control strength. Then, participants were led to the sports hall where they put on a harness and received instructions for the HRSST: They were instructed to climb to the top of the wall top-rope secured $(12 \mathrm{~m})$. Having reached the top of the wall, participants reported their current anxiety state. Then the belayer explained that they had to "jump into the rope", ${ }^{1}$ resulting in a fall of about $2-3 \mathrm{~m}$. The instruction was to jump backward, not to touch the rope, and to land with both feet simultaneously on the wall. The participants were instructed to choose the moment of the jump themselves. The jump (or fall) was extended by the belayer by loosening the rope in the moment of the fall. If participants displayed at least three of five previously defined abort criterions at this point (i.e., shaking of the legs, slowdown and solidification of movements, cramping, loud and panting breathing and repeated asking), the study was stopped. After the jump, participants were lowered by the belayer.

Finally, the participants were led to the third room where the remaining measurements were taken. At the moment of the participant's jump into the rope, the investigator started

\footnotetext{
1 "Jumping into the rope" is a term belonging to "fall training". Jumping and falling often is experienced as exciting by beginners. This circumstance was used for stress induction.
}

a digital stopwatch (CASIO, HS-3V-1RET) so that, after the jump, four salivary samples could be taken with 10-min intervals (t3-t6). Moreover, at each time point, participants again filled out questionnaires regarding state anxiety (WAI-S; Ehrlenspiel et al., 2009). After the final measurement (i.e., at t6), participants completed questionnaires to assess both personality traits (e.g., Mindfulness: Kentucky Inventory for Mindfulness Skills Short; Höfling et al., 2011; Sensation Seeking: SSS-V; Zuckerman, 1994; German version: Beauducel et al., 2003). After the study, participants were thanked, compensated, and fully debriefed.

\section{Measures \\ Predictors}

\section{Sensation seeking}

Sensation seeking was assessed with the Sensation Seeking Scale V (SSS-V; Zuckerman, 1994, German version: Beauducel et al., 2003). The instrument consists of 40 items in a forced-choice format (e.g., "I would like to try to surf". vs. "I would not like to try to surf".) of which ten items can be allocated to one of the following for facets, respectively: 1. Thrill and Adventure Seeking (TAS): Search for danger and adventures, 2. Experience Seeking (ES): Search for experiences through a non-conformist lifestyle, 3. Disinhibition (DIS): Tendency for disinhibition in social situations, 4. Boredom Susceptibility (BS): Aversion to repetition and routines. Besides the separate scores for each facet, a total score varying between $\min =0$ and $\max =40$ can be calculated. Based on the current sample, the questionnaire showed satisfactory to good internal consistency, with values similar to those reported for the norm sample (i.e., total scale: $\alpha=0.82$; subscales between 0.64 and 0.81 ; cf. Beauducel et al., 2003).

\section{Mindfulness}

Mindfulness was measured using the Kentucky Inventory for Mindfulness Skills Short (KIMS-D Short; German version: 
Höfling et al., 2011). The 20 items of the short version are to be answered on a five-point Likert-type scale (ranging from $1=$ never or seldom to $5=$ very often or always) with participants rating to which extent the statements applied to them. The items can be allocated to the subscales observation of external phenomena (obs-ext), observation of internal phenomena (obsint), describing (des), acting mindfully $(A M)$ and accepting without rating ( $A W R$; Höfling et al., 2011). An example item for the $A M$ scale is "I judge whether my thoughts are good or bad". The five scales were combined to an index for mindfulness (in the following called mindfulness index). For mindfulness measured with the KIMS-D Short (Höfling et al., 2011), all reliabilities were satisfactory to good (i.e., $\alpha=0.74$ for observing to $\alpha=0.85$ for describing) and comparable to norm-values reported in the literature (i.e., $\alpha=0.70-0.89$; Höfling et al., 2011).

\section{Psychological Stress Response State anxiety}

Participants' psychological response to the stressor was assessed using two measures of state anxiety: Firstly, it was assessed at two measurement points $(t 1, t 3)$, using the German questionnaire Wettkampf-Angst-Inventar-State (WAI-S; Ehrlenspiel et al., 2009). The WAI-S consists of 12 items (four-point scale, from $0=$ "not at all" to 3 = "extremely") and the subscales somatic anxiety (som), cognitive anxiety (cog) and confidence (conf). An example item for the somatic anxiety subscale is "In the present moment. . my heart throbs". The internal consistencies of the WAI-S were found to be clearly (at $t 1$, before the stress induction) and slightly (at $t 3$, after the stress induction) lower in the present sample (between $\alpha_{\operatorname{cog}}=0.52$ and $\alpha_{\text {conf }}=0.79$ ), compared to the norm sample (Ehrlenspiel et al., 2009: between $\alpha_{\operatorname{cog}}=0.79$ and $\left.\alpha_{\text {conf }}=0.82\right)$. Secondly, state anxiety was assessed at three measurement points $(t 1, t 2, t 3)$ using an anxiety thermometer (Houtman and Bakker, 1989). The anxiety thermometer captures the current feelings of anxiety by one item asking the question "How do you rate your current feelings of anxiety?" on a 10$\mathrm{cm}$ visual analog scale, ranging from $0=$ no anxiety at all to 10 = extreme anxiety. Houtman and Bakker (1989) report testretest reliabilities of $0.60-0.70$.

In this experiment, we applied two different measures of state anxiety at the same time. The questionnaire WAI-S permits with its three subscales a more detailed view of the facets of anxiety while at the same time taking more time to fill it out. The one-item anxiety thermometer promised a weaker reliability, but a better handling during the climbing task (see Nieuwenhuys et al., 2008).

\section{Physiological Stress Response Cortisol}

The physiological stress response was assessed repeatedly using the cortisol concentration in saliva. Participants' samples were collected using Salivette Blue ${ }^{\circledR}$ Device (Sarstedt GmbH, Nümbrecht). Thereby, participants chewed on a synthetic swab for $1 \mathrm{~min}$. As the cortisol peaks about $20 \mathrm{~min}$ after experiencing the stressor (the jump) (Kirschbaum and Hellhammer, 2000; Campbell and Ehlert, 2012), saliva samples were taken five times in 10-min intervals after the stress induction (10, 20,
30, and $40 \mathrm{~min}$ after the jump). The measurement point of interest was $t 4,20 \mathrm{~min}$ after the jump. Additionally, the return to baseline levels could be assessed from the later measurement points. The saliva samples were stored at $-20^{\circ} \mathrm{C}$ until analyses. The biochemical analysis of the samples was conducted by the steroid laboratory of the Steroid Laboratory, University Hospital Heidelberg. After thawing, the samples were centrifuged at $3000 \mathrm{rpm}$ for $5 \mathrm{~min}$ which resulted in a clear supernatant of low viscosity. Salivary concentrations were determined using chemiluminescence immunoassay with high sensitivity (IBL International, Hamburg, Germany). The intra- and inter-assay coefficients for cortisol, which express the precision or repeatability of immunoassay test results, were good (i.e., below 8\%; cf. Schultheiss and Stanton, 2009).

\section{Control Variables}

\section{Trait anxiety}

The control variable trait anxiety was measured with the German questionnaire Wettkampf-Angst-Inventar-Trait (WAI-T; Brand et al., 2009). The inventory consists of 12 items (four-point scale, from $0=$ "not at all" to 3 = "extremely"). The 12 items can be allocated to the scales somatic anxiety (som), cognitive anxiety (cog) and concentration difficulties (conc). The items of the WAI-T are similar to those of the WAI-S, however, the introductory formulation "In the present moment. .." is replaced by "Before sporting challenges...". In line with Smith et al.'s (1990) Sport Anxiety Scale - on which the WAI-T was based and to optimize statistical power of our regression models, WAI$\mathrm{T}$ subscales were combined to arrive at a single trait anxiety score for each individual (in the following called "WAI-T index"). ${ }^{2}$ In general, internal consistency of the WAI-T was acceptable to good (with $\alpha$ s between 0.60 and 0.82 ) and comparable to original values reported by Brand et al. (2009); i.e., with as between 0.77 and 0.81 ).

\section{Data Processing and Statistical Analyses}

Initially, the data of all variables were analyzed to detect any missing and extreme values. Because missing values only occurred occasionally $(\leq 5 \%)$ and unsystematically, they were replaced using the expectation maximization (EM) method (Wirtz, 2004; Tabachnick and Fidell, 2007). Concerning cortisol, missing values were replaced using multiple imputations. Beforehand, necessary conditions were checked separately for each group using missing-completely-at-random (MCAR) tests (Tabachnick and Fidell, 2007). To identify extreme values, boxplots were created separately for experimental groups (depletion and non-depletion) and measurement points. Tukeyfar-out was chosen as a criterion for extreme values: Values which are more than the triple interquartile range above/under the $75 \% / 25 \%$ quartile were identified as extreme values (Tabachnick and Fidell, 2007). Concerning cortisol, the exclusion criterion

\footnotetext{
2 "As a robustness check, all analyses were also performed with the WAI-T subscales as covariates. With regards to the associations between sensation seeking, mindfulness and our outcome measures, results for these analyses were highly similar to the reported analyses and showed the same significant and nonsignificant effects. Detailed analysis reports can be obtained from the first author upon request".
} 
was a distance of \pm 3 SDs above/under the average group value (Adam and Kumari, 2009).

After the data had been prepared, it was checked for normal distribution as a necessary condition for the following arithmetical analyses. As the Kolmogorov-Smirnov test easily detects a violation of the normal distribution in big samples, additionally, histograms were used. If the KolmogorovSmirnov test was found to be significant, analyses were conducted nonetheless because of its high sensitivity and nonparametric relations were calculated on a descriptive statistical level additionally.

To identify the covariates, the dependent variables (i.e., state anxiety and cortisol) were correlated with possible covariates, including age, fitness, previous climbing experience, trait anxiety and experimental condition (ego depletion vs. no ego depletion). In addition, as a possible covariate for cortisol, starting time (time of day) was coded as a variable to control for the influence of the decrease in cortisol during the day. Beforehand, the covariate was adjusted for extreme values following the procedure described above.

Cortisol values were checked substantially and arithmetically for plausibility. To display the change in the response to the stressor, different characteristic values were calculated. Firstly, the increase from baseline to the jump was calculated by taking the difference between $t 1$ and $t 4$ (i.e., 20 min after the jump), after values had been approximated to the normal distribution using the box-cox-transformation (Miller and Plessow, 2013). In addition, two different versions of the area under the curve (AUC) were calculated, the area under the curve with respect to ground (AUCg) and the area under the curve with respect to increase (AUCi; Pruessner et al., 2003). The AUCg is considered as an indicator for the absolute cortisol concentration over time, mapping the total area of trapezes between the measurement points. For the AUCi, the area between the first measurement point (baseline) and the zero point is subtracted from AUCg. $\mathrm{AUC}_{\mathrm{i}}$ thus represents the change in cortisol over time compared to the baseline. If the value is positive, an increase occurs. If the AUCi value is negative, cortisol decreases.

To test hypotheses 1 and 2, (partial) correlations between the independent and the dependent variables (while controlling for covariates) were calculated. The correlations are based on $z$-standardized variables.

To test hypotheses 3 and 4, hierarchical regressions were calculated, using SS and mindfulness (plus the covariates) as predictors and anxiety (WAI-S at $t 1$ and $t 3$, anxiety thermometer at $t 1, t 2$, and $t 3$ ) and cortisol (AUCg, AUCi, increase and cortisol at $t 4$ ) as criteria. Doing so, covariates were entered in step 1, followed by sensation seeking in step 2 and mindfulness in step 3. To increase the interpretability of the residues, predictors were grand-mean centered (Field, 2009). The covariates age and previous climbing experience (in hours) were not centered because a useful unit already existed.

The assessment of statistical significance followed conventional criteria. A probability of $p<0.10$ was considered as marginally significant, of $p<0.05$ as significant, of $p<0.01$ as highly significant and of $p<0.001$ as extremely significant. IBM SPSS Statistics 24 was used for all statistical analyses.

\section{RESULTS}

\section{Data Preparation}

Initially, the cortisol values were checked for plausibility: All values that were located outside the area that can be assessed for analyses using the assay (0.414-41.4 nmol/l) were excluded. This exclusion affected three participants for $t 1$ and two participants for $t 3$. Missing values were replaced using multiple imputation: This affected three participants for $t 1$ and four participants for t3. In total, seven participants had to be excluded from the study because of content-related reasons or an extreme value at the third measurement point.

As there were no significant correlations between the experimental condition (ego depletion vs. no ego depletion) and the criteria (anxiety/cortisol), this potential covariate was not included into the regression analyses. Based on the significant correlations (see Supplementary Material 1), the covariates age, fitness state and climbing frequency as well as the WAI-T index were included in the respective analyses.

\section{Time Course Analyses}

To indicate the overall effectiveness of the stress manipulation (i.e., regardless of sensation seeking and mindfulness), differences in state anxiety and cortisol before and after the climb (see Table 1) were compared across all participants using paired $t$-tests. In response to the stressor, on a psychological level, anxiety (as indexed by the anxiety thermometer) increased significantly over time (baseline $t 1$ compared to $t 2$ on top of the climbing wall) by an average of 2.73 points [i.e., 10point scale; $S D=2.32 ; \operatorname{Min}=-1, \operatorname{Max}=9 ; t(80)=-10.73$, $p<0.001 ; d=0.95]$. On a physiological level, salivary cortisol concentrations ( $t 1$ compared to $t 4$ ) showed a slight but nonsignificant increase by an average of $0.79 \mathrm{nmol} / 1$ [ $S D=4.85$; Min $=-16.60, \operatorname{Max}=12.22 ; t(80)=-1.51, p=0.13 ; d=0.17]$.

\section{Correlations (Hypotheses 1 and 2)}

As appears from Table 2, measures of sensation seeking (SS scale) and mindfulness (KIMS) were not significantly correlated, $r(82)=0.11, p=0.33$.

Regarding Hypothesis 1a, sensation seeking (SS total score) did not significantly correlate with any of the anxiety measures at baseline (i.e., $t 1$; see Table 3). After the climb, however, sensation seeking was negatively correlated with the WAI-S somatic scale

TABLE 1 | Time course analysis.

\begin{tabular}{|c|c|c|c|c|}
\hline Variables & $M(S D)$ at $t 1$ & $M(S D)$ at $t 2$ & $M(S D)$ at $t 3$ & $M(S D)$ at $t 4$ \\
\hline WAI-S som & $1.48(0.44)$ & - & $1.91(0.49)$ & - \\
\hline WAI-S cog & $1.34(0.36)$ & - & $1.26(0.33)$ & - \\
\hline WAI-S conf & $2.94(0.48)$ & - & $3.03(0.62)$ & - \\
\hline $\begin{array}{l}\text { Anxiety } \\
\text { thermometer }\end{array}$ & $0.8(0.8)$ & $3.5(2.4)$ & $1.3(1.6)$ & - \\
\hline $\begin{array}{l}\text { Salivary cortisol } \\
\text { (in } \mathrm{nmol} / \mathrm{l} \text { ) }\end{array}$ & $9.42(9.96)$ & - & - & $10.21(7.40)$ \\
\hline
\end{tabular}

WAI-S, Wettkampf-Angst-Inventar-State; WAI-S som, WAI-S somatic anxiety; WAI$S$ cog, WAI-S cognitive anxiety; WAl-S conf, WAI-S confidence. 
TABLE 2 | (Partial) correlations of the independent variables.

\begin{tabular}{|c|c|c|c|c|c|c|c|c|c|c|c|}
\hline Variables & 1 & 2 & 3 & 4 & 5 & 6 & 7 & 8 & 9 & 10 & 11 \\
\hline 1. SS total & - & $0.67^{* * *}$ & $0.73^{* * *}$ & $0.74^{* * *}$ & $0.63^{* * *}$ & 0.11 & $0.23^{*}$ & 0.10 & 0.00 & 0.03 & -0.05 \\
\hline 2. SS BS & - & - & $0.32 * *$ & $0.38^{* * *}$ & 0.17 & 0.11 & $0.22^{*}$ & 0.09 & 0.03 & 0.01 & -0.07 \\
\hline 3. SS Dis & - & - & - & $0.34^{* *}$ & $0.22^{*}$ & -0.03 & 0.13 & -0.01 & -0.11 & -0.01 & -0.06 \\
\hline 4. SS ES & - & - & - & - & $0.42^{* * *}$ & 0.17 & 0.20 & 0.14 & 0.04 & 0.00 & 0.06 \\
\hline 5. SS TAS & - & - & - & - & - & 0.08 & 0.08 & 0.08 & 0.06 & 0.09 & -0.04 \\
\hline 6. KIMS index & - & - & - & - & - & - & $0.66^{* * *}$ & $0.53^{* * *}$ & $0.45^{* * *}$ & $0.47^{* * *}$ & $0.48^{* * *}$ \\
\hline 7. KIMS obs-ext & - & - & - & - & - & - & - & $0.52^{* * *}$ & -0.09 & -0.06 & 0.03 \\
\hline 8. KIMS obs-int & - & - & - & - & - & - & - & - & -0.09 & $0.25^{*}$ & 0.08 \\
\hline 9. KIMS des & - & - & - & - & - & - & - & - & - & 0.09 & $0.31^{* *}$ \\
\hline 10. KIMS am & - & - & - & - & - & - & - & - & - & - & -0.05 \\
\hline 11. KIMS awr & - & - & - & - & - & - & - & - & - & - & - \\
\hline
\end{tabular}

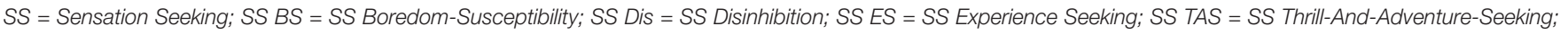

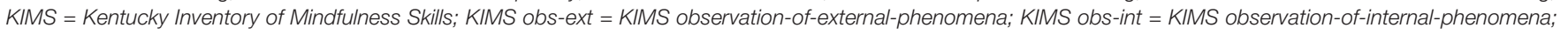
KIMS des = KIMS describing; KIMS am = KIMS acting-mindfully; KIMS awr = KIMS accepting-without-rating. ${ }^{*} p<0.05 .{ }^{* *} p<0.01 .{ }^{* * *} p<0.001$.

at $t 3[r(82)=-0.22, p=0.01]$ and with the WAI-S cognitive scale at $t 3[r(82)=-31, p=0.01]$. No significant correlations of the SS total score were found with the WAI-S somatic scale at $t 3$ as well as with the anxiety thermometer at $t 2$ or $t 3$. Regarding Hypothesis $1 \mathrm{~b}$, no relationship was observed between sensation seeking and cortisol responses (see Table 3 ).

Regarding Hypothesis 2a, mindfulness (KIMS index) was negatively correlated with one component of anxiety (i.e., WAI-S confidence scale) at baseline [i.e., $t 1 ; r(92)=-22, p=0.02$ ]. No other significant correlations were found. Regarding Hypothesis $2 \mathrm{~b}$, no relationship was observed between mindfulness and cortisol responses (see Table 3).

In addition to the above, various correlations were found between psychological and physiological responses (see Table 4): AUCi correlated significantly positively with the anxiety thermometer at $t 1[r(79)=0.27, p=0.02]$ and the anxiety thermometer at $t 2[r(79)=0.27, p=0.02]$. The cortisol value at $t 4$ $[r(79)=0.27, p=0.02]$ and the increase in cortisol $[r(79)=0.23$, $p=0.04$ ] each displayed a significant positive correlation with the anxiety thermometer at $t 2$. No significant associations were found between the remaining dependent variables (see Table 4). At a physiological data level, no significant relationship was found between AUCi and AUCg $(r=0.10, p=0.40)$. The reason may be that, as argued by Pruessner et al. (2003), the variables mirror differential aspects of the physiological response. Moreover, AUCg was not correlated significantly with the increase in cortisol $[r(79)=0.11, p=0.33]$ but displayed a significant positive correlation with the cortisol value at $t 4$ $[r(79)=0.84, p<0.001]$.

\section{Hierarchical Regression Analyses (Hypotheses 3 and 4)}

Regarding Hypothesis 3a, after controlling for covariates, sensation seeking (SS total score) did not predict any of the WAI-S anxiety measures at baseline (i.e., $t 1$ ), but was found to be a marginally significant predictor of anxiety as measured with the anxiety thermometer (see Table 5). After the climb (i.e., at t3), sensation seeking (SS total score) again marginally predicted anxiety as measured with the anxiety thermometer and could explain $26.8 \%$ of the total variance in WAI-S somatic $\left[R^{2}=0.27, \Delta R^{2}=0.07\right.$, $F(3,84)=11.60, p<0.001]$ and $21.6 \%$ of the variance in WAI-S cognitive $\left[R^{2}=0.22, \Delta R^{2}=0.08, F(3,84)=24.50\right.$, $p<0.001]$. For the confidence component, SS was found to be a marginally significant predictor (see Table 5). Regarding Hypothesis 3b, no significant associations were observed between sensation seeking (SS total score) and any of the physiological variables (i.e., AUCg, AUCi, increase in cortisol and cortisol at $t 4)$.

Regarding Hypothesis 4a, after controlling for covariates and sensation seeking, mindfulness (KIMS index) significantly predicted WAI-S confidence at baseline $[\beta=0.13, t(83)=2.11$, $p=0.04]$. Together, the three predictors could explain $22.3 \%$ of the total variance $\left[R^{2}=0.22, \Delta R^{2}=0.04, F(3,84)=8.03\right.$, $p<0.001]$. After the climb (i.e., at $t 3$ ), mindfulness was found to be a marginally significant predictor of WAI-S confidence, but not for the two other components of the WAI$S$ (Table 5). Mindfulness did not predict anxiety as measured with the anxiety thermometers at any measurement point (see Table 5). Regarding Hypothesis 4b, no significant associations were observed between mindfulness (KIMS index) and any of the physiological variables (i.e., AUCg, AUCi, increase in cortisol and cortisol at $t 4)$.

\section{DISCUSSION}

The current study investigated whether sensation seeking and mindfulness affect individuals' psychological (anxiety) and physiological (cortisol) response to a sport-specific stressor: The HRSST. It was hypothesized that both sensation seeking and mindfulness would negatively correlate with anxiety ( $\mathrm{H} 1 \mathrm{a}$ and $\mathrm{H} 2 \mathrm{a}$ ) and cortisol ( $\mathrm{H} 1 \mathrm{~b}$ and $\mathrm{H} 2 \mathrm{~b}$ ), that sensation seeking would be a significant predictor of anxiety and cortisol in response to the HRSST (H3a and $\mathrm{H} 3 \mathrm{~b}$ ), and that - beyond sensation seeking mindfulness would explain additional variance in anxiety and cortisol ( $\mathrm{H} 4 \mathrm{a}$ and $\mathrm{H} 4 \mathrm{~b})$. 


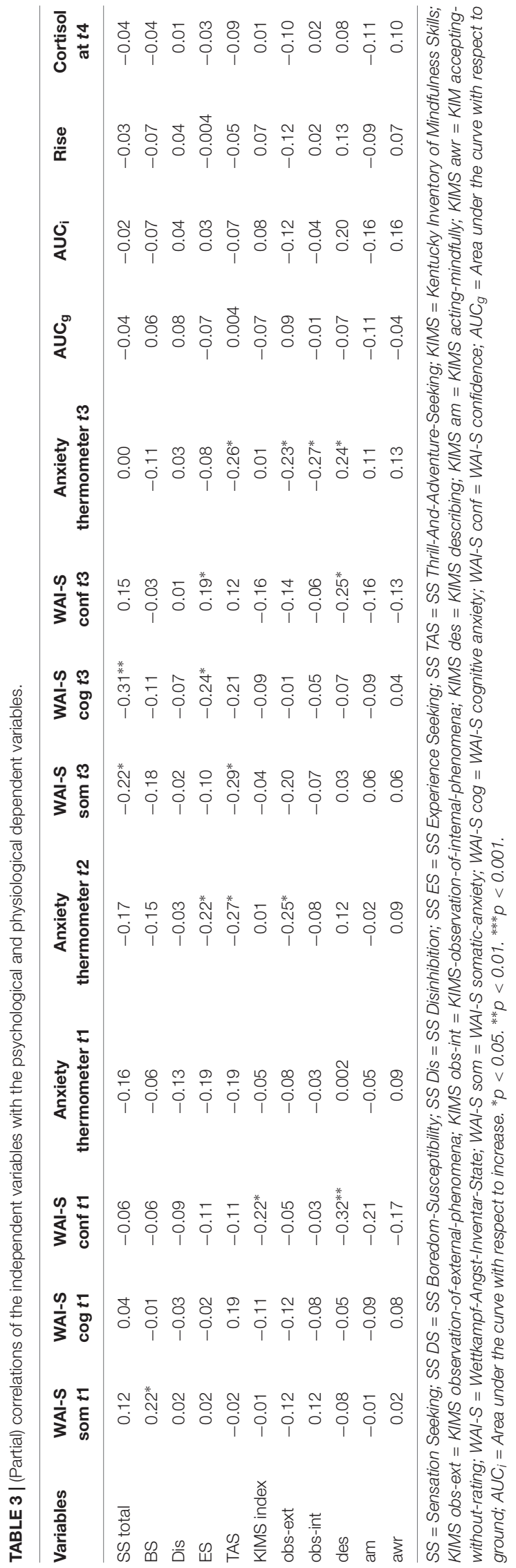

\section{Psychological Response to the HRSST Sensation Seeking}

Our findings partly confirm hypotheses $\mathrm{Hla}$ and $\mathrm{H} 3 \mathrm{a}$ that sensation seeking (SS) would be associated with and significantly predict participants' psychological response to the HRSST. At $t 3$, we found a significant negative relationship between SS and two of the subscales of state anxiety (the somatic and the cognitive component). In the corresponding regression analyses (i.e., predicting both anxiety components at $t 3$ ) - and after controlling for covariates - SS explained a significant proportion of variance, with individuals scoring high on SS exhibiting lower levels of anxiety. As became apparent from the analyses of subscales, the observed effects are likely driven by the TAS component and the ES component of SS (see Table 3). This observation is in line with a study conducted by Breivik (1999a), in which the difference in SS between high risk sports athletes and sport science students was also caused by TAS and ES. As such, TAS and ES appear to be essential components of SS and its effect on individuals' psychological response to risk-full sport situations. The current results are in line with the psychobiological multilevel theory (Zuckerman, 1994) and show that HSS differ from LSS in their psychological response to an unexpected stimulus, in this case the jump into the rope.

Contradicting H1a and H3a, SS was neither significantly associated with nor significantly predicted the confidence component of state anxiety. Whilst speculative, this may relate to the nature of the current task (i.e., a wall climb followed by a so-called jump in the rope), with which (a) participants had very little or no experience; and which (b), allowed participants little control over the course of action. Potentially, with higher levels of task experience or in tasks that allow more control, sensation seeking may also boost self-confidence and further contribute to the positive appraisal of high-risk performance environments that is characteristic of HSS. Future research aiming to investigate this matter may find Jones' (1995) control model of anxiety which distinguishes between intensity and direction (i.e., positive vs. negative) of the anxiety response - to be a useful framework.

Matching the effects observed with the WAI-S (somatic and cognitive anxiety subscale), SS was found to explain a significant proportion of variance in participants' scores on the anxiety thermometer at $t 3$. Unexpectedly, the effect of SS on anxiety thermometer scores at $t 2$ failed to reach significance $(p=0.11$, see Table 5). With the effect of SS on anxiety being generally small (see Table 5), one explanation for the absence of this effect may be that - being a one-item measure - the anxiety thermometer may simply not have been sensitive enough to detect a statistically significant difference. Indeed, a posteriori power analyses with G-Power (Faul et al., 2009) - based on the current sample and analyses and with effect sizes as reported in Table 5 - indicate that statistical power for the anxiety thermometer at $t 2$ was insufficient to detect a small effect (i.e., with power $=0.67$ ), whereas power was sufficient (i.e., $>0.85$ ) for all other dependent variables.

\section{Mindfulness}

Our findings largely contradict hypotheses $\mathrm{H} 2 \mathrm{a}$ and $\mathrm{H} 4 \mathrm{a}$ in that mindfulness was neither significantly associated with nor significantly predicted anxiety in response to the HRSST. 
TABLE 4 | (Partial) correlations of the psychological and physiological dependent variables.

\begin{tabular}{|c|c|c|c|c|c|c|c|c|c|c|c|c|c|}
\hline Variables & 1 & 2 & 3 & 4 & 5 & 6 & 7 & 8 & 9 & 10 & 11 & 12 & 13 \\
\hline 1. WAI-S som $t 1$ & - & $0.26^{*}$ & 0.08 & $0.28^{* *}$ & -0.01 & 0.21 & $0.23^{*}$ & 0.03 & 0.05 & 0.00 & 0.04 & 0.03 & 0.05 \\
\hline 2. WAI-S $\operatorname{cog} t 1$ & - & - & $-0.22 *$ & 0.15 & 0.04 & 0.01 & $0.34^{* *}$ & $0.49 * * *$ & 0.09 & 0.13 & 0.01 & 0.00 & 0.06 \\
\hline 3. WAI-S conf $t 1$ & - & - & - & $-0.28^{*}$ & 0.12 & 0.04 & -0.17 & $0.49^{* * *}$ & $0.29^{* *}$ & 0.16 & -0.06 & -0.06 & 0.03 \\
\hline 4. Anxiety thermometer $t_{1}$ & - & - & - & - & $0.24^{*}$ & 0.21 & $0.26^{*}$ & $-0.21^{*}$ & $0.43^{*}$ & 0.04 & $0.27^{*}$ & 0.22 & 0.19 \\
\hline 5. Anxiety thermometer $t_{2}$ & - & - & - & - & - & $0.49^{* * *}$ & $0.27^{* *}$ & 0.01 & $0.61^{* *}$ & 0.19 & $0.27^{*}$ & $0.23^{*}$ & $0.27^{*}$ \\
\hline 6. WAI-S som t3 & - & - & - & - & - & - & $0.39^{* * *}$ & -0.10 & $0.56^{* *}$ & -0.04 & 0.15 & 0.13 & 0.10 \\
\hline 7. WAI-S $\operatorname{cog} t 3$ & - & - & - & - & - & - & - & $-0.38^{* * *}$ & $0.54^{* *}$ & -0.12 & 0.21 & 0.16 & 0.13 \\
\hline 8. WAI-S conf $t 3$ & - & - & - & - & - & - & - & - & $0.32^{* *}$ & 0.11 & -0.06 & -0.08 & -0.00 \\
\hline 9. Anxiety thermometer $t_{3}$ & - & - & - & - & - & - & - & - & - & & & $0.35^{* *}$ & 0.18 \\
\hline 10. $\mathrm{AUC}_{\mathrm{g}}$ & - & - & - & - & - & - & - & - & - & - & 0.10 & 0.10 & $0.84^{* * *}$ \\
\hline 11. $A \cup C_{i}$ & - & - & - & - & - & - & - & - & - & - & - & $0.88^{* * *}$ & $0.86^{* * *}$ \\
\hline 12. rise $t 1-t 4$ & - & - & - & - & - & - & - & - & - & - & - & - & $0.89^{* * *}$ \\
\hline 13. Cortisol t4 & - & - & - & - & - & - & - & - & - & - & - & - & - \\
\hline
\end{tabular}

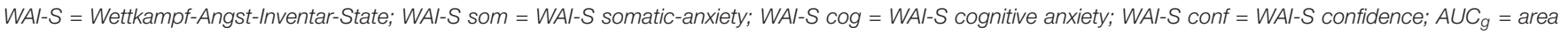
under the curve with respect to ground; $A \cup C=$ area under the curve with respect to increase. ${ }^{*} p<0.05$. ${ }^{* *} p<0.01$. ${ }^{* * *} p<0.001$.

TABLE 5 | Hierarchical regressions of the dependent variables on SS and KIMS and the respective covariates.

\begin{tabular}{|c|c|c|c|c|c|}
\hline $\begin{array}{l}\text { Dependent } \\
\text { variable }\end{array}$ & $\begin{array}{l}\text { Covariate(s), } \\
\text { independent } \\
\text { variables }\end{array}$ & $\begin{array}{c}\text { Standardized } \\
\text { beta }\end{array}$ & $\Delta R^{2}$ & $P$ & $f^{2}$ \\
\hline \multirow[t]{3}{*}{ WAIS som $t 1$} & WAIT index & $0.46^{* * *}$ & 0.22 & 0.00 & 0.28 \\
\hline & SS-total & 0.02 & 0.00 & 0.85 & 0.00 \\
\hline & KIMS-index & 0.01 & 0.00 & 0.94 & 0.00 \\
\hline \multirow[t]{3}{*}{ WAIS $\operatorname{cog} t 1$} & WAIT-index & $0.49^{* * *}$ & 0.22 & 0.00 & 0.28 \\
\hline & SS-total & 0.04 & 0.00 & 0.68 & 0.00 \\
\hline & KIMS-index & -0.06 & 0.01 & 0.47 & 0.01 \\
\hline \multirow[t]{3}{*}{ WAIS conf $t 1$} & WAIT-index & $0.26 * * *$ & 0.18 & 0.00 & 0.22 \\
\hline & SS-total & 0.04 & 0.00 & 0.87 & 0.00 \\
\hline & KIMS-index & $0.13^{*}$ & 0.04 & 0.04 & 0.04 \\
\hline Anxiety & WAIT-index & $0.39 * * *$ & 0.13 & 0.00 & 0.15 \\
\hline \multirow[t]{2}{*}{ thermometer $t 1$} & SS-total & -0.18 & 0.03 & 0.08 & 0.03 \\
\hline & KIMS-index & -0.00 & 0.00 & 0.98 & 0.00 \\
\hline Anxiety & Climbing & $-0.25^{*}$ & 0.09 & 0.02 & 0.10 \\
\hline \multirow[t]{2}{*}{ thermometer $t 2$} & SS-total & -0.17 & 0.03 & 0.11 & 0.03 \\
\hline & KIMS-index & -0.09 & 0.01 & 0.41 & 0.01 \\
\hline \multirow[t]{4}{*}{ WAIS som $t 3$} & WAIT-index & $0.44^{* * *}$ & 0.17 & 0.00 & 0.20 \\
\hline & Climbing & -0.17 & 0.05 & 0.09 & 0.05 \\
\hline & SS-total & $-0.27^{* *}$ & 0.07 & 0.01 & 0.08 \\
\hline & KIMS-index & -0.02 & 0.00 & 0.84 & 0.00 \\
\hline \multirow[t]{3}{*}{ WAIS $\operatorname{cog} t 3$} & WAIT-index & $0.45^{* * *}$ & 0.15 & 0.00 & 0.18 \\
\hline & SS-total & $-0.29^{* *}$ & 0.08 & 0.00 & 0.09 \\
\hline & KIMS-index & -0.02 & 0.00 & 0.86 & 0.00 \\
\hline \multirow[t]{3}{*}{ WAIS conf $t 3$} & WAIT-index & $0.32^{* *}$ & 0.12 & 0.00 & 0.14 \\
\hline & SS-total & -0.19 & 0.03 & 0.07 & 0.03 \\
\hline & KIMS-index & 0.18 & 0.03 & 0.09 & 0.03 \\
\hline Anxiety & WAIT-index & $0.40^{* * *}$ & 0.13 & 0.00 & 0.15 \\
\hline \multirow[t]{3}{*}{ thermometer $t 3$} & Climbing & -0.18 & 0.06 & 0.08 & 0.06 \\
\hline & SS-total & -0.20 & 0.04 & 0.05 & 0.04 \\
\hline & KIMS-index & -0.09 & 0.01 & 0.37 & 0.01 \\
\hline
\end{tabular}

SS = Sensation Seeking; WAI-S = Wettkampf-Angst-Inventar-State; WAI-S som = WAI-S somatic-anxiety; WAI-S cog = WAI-S cognitive anxiety; WAI-S conf = WAI-S confidence; KIMS = Kentucky Inventory of Mindfulness Skills. ${ }^{*} p<0.05$. ${ }^{* *} p<0.01 .{ }^{* * *} p<0.001$. A posteriori power analyses: small ( $\left.f^{2}=0.02\right)$, medium $\left(f^{2}=0.15\right)$, large effects $\left(f^{2}=0.35\right) ; \alpha$ err prob $<0.05$.
The only significant associations that were observed regarded the WAI-S confidence subscale at baseline (see Tables 3, 5), indicating that mindful individuals tended to show slightly lower baseline levels of confidence. Note however, that baseline levels of confidence were generally positive and showed little betweensubject variation (see Table 1). As such, the observed effect is likely to be of low clinical significance. Analyses of subscales (see Table 3) suggest that the observed effect is likely driven by the KIMS subscale "describing," which was negatively correlated with the confidence component of state anxiety at $t 1$ as well as at t3. Participants with a stronger tendency to (explicitly) describe phenomena in their surroundings reported lower confidence before and after the jump.

The general absence of significant effects regarding mindfulness may be explained by the correlational design of the current study and, potentially, insufficient variability in trait mindfulness (as measured with the KIMS), as well as the fact that the current study deliberately examined effects of mindfulness over and above effects of sensation seeking. As can be seen in Table 5, in case of significant effects, substantial variance in outcome measures was often explained by covariates (e.g., trait anxiety) and sensation seeking, leaving little room for mindfulness to make an additional impact. Still, narrative research from extreme sports (Brymer and Schweitzer, 2013; Arijs et al., 2017; Houge Mackenzie and Brymer, 2018) as well as theoretical explanations from other contexts than sports (Mindfulness Stress Buffering Account; Creswell and Lindsay, 2014; Creswell et al., 2019) suggest a link between trait mindfulness and state anxiety. Future studies are advised to consider effects across a broader range of mindfulness, either by contrasting extremes or by implementing tailored mindfulness interventions.

\section{Physiological Response to the HRSST Sensation Seeking}

Our findings contradict hypotheses $\mathrm{H} 1 \mathrm{~b}$ and $\mathrm{H} 3 \mathrm{~b}$, indicating no significant association between SS and salivary cortisol in response to the HRSST. This lack of association is surprising, 
as a previous study using the same stress induction protocol (Frenkel et al., 2018b) showed that HSS compared to LSS showed significantly smaller cortisol responses. In the broader literature, however, an apparent dissociation between physiological and psychological stress responses is not uncommon (Breivik, 1999a; Kudielka et al., 2009; Campbell and Ehlert, 2012). A review including 49 studies using the Trier Social Stress Test as a psychosocial stressor detected this mismatch in $75 \%$ of the studies (Campbell and Ehlert, 2012). This controversy of the results is explained, among others, by inter-individual differences in the degree of psycho-physiological correspondence, or possible mediating factors.

In explaining the observed null-finding, it is important to consider that the current sample consisted exclusively of highly fit and physically active sport science students, whose selfreported fitness level averaged around $70.98(S D=18.29)$ on a $0-100$ scale. In line with the stressor adaptation hypothesis (CSA hypothesis; Sothmann et al., 1996), which suggests that adaptation to physical stress (e.g., following regular physical exercise) may transfer to include other stressors, several groups of researchers have found reduced psychological and physiological responses to psychological stress in physically active individuals (Klaperski et al., 2013; Zschucke et al., 2015). Matching these observations, the current study showed that - regardless of sensation seeking and mindfulness - increases in anxiety and salivary cortisol following the HRSST were small and, in case of cortisol, non-significant (see "Time course analyses" in the Results section). Addressing this issue, future studies on risk sport-specific stress which examine a highly physically active population, are advised to consider additional means to further intensify the stress protocol.

\section{Mindfulness}

Our findings contradict hypotheses $\mathrm{H} 2 \mathrm{~b}$ and $\mathrm{H} 4 \mathrm{~b}$, indicating no significant associations between mindfulness and physiological stress response measured by salivary cortisol. As with sensation seeking, this null-finding is likely explained by the non-significant increase in cortisol following the HRSST. In addition to increasing the intensity of the stressor, future studies may consider to examine effects in the context of mindfulnessand acceptance-based interventions (e.g., Gardner and Moore, 2004, 2017; Birrer et al., 2012; Frenkel et al., 2018a; Josefsson et al., 2019) as opposed to examining (small) inter-individual differences in trait mindfulness. Although mindfulness training has a long standing tradition in applied sports psychology (Gardner and Moore, 2004, 2017), only few evidence based intervention studies have examined the effects of mindfulness practice on physiological and psychological performance surrogates or on performance outcomes in sports (Bühlmayer et al., 2017; Hoja et al., 2018). One intervention study that did investigate effects of a mindfulness intervention on HPA axis activation reported decreased salivary cortisol levels following mindfulness (John et al., 2011). In this regard, potential implications remain promising.

\section{Potential, Limitations, and Outlook}

To our knowledge, the current study is the first to examine the effects of sensation seeking and trait mindfulness on psychological and physiological responses to a standardized risk sport-specific stressor. A strength of the current study is the application of an experimentally controlled nature and external validity of the stressor, the HRSST, which allows robust examinations of stress responses and realistically mimics stressful situations in high risk sports. Results from the current study, as well as previous work (e.g., Frenkel et al., 2018b) indicate that the HRSST induces a consistent psychological response, which is characterized by robust increases in self-reported state anxiety. On the other hand, physiological responses to the HRSST have been more inconsistent. Salivary cortisol significantly increased after the HRSST in Frenkel et al.'s (2018b) initial validation study, but did not significantly increase in the current study. It is therefore important to further develop the paradigm so that an increase in cortisol can be reliably induced - also in highly fit and physically active populations. Potential considerations include prolonging the task or adding additional (external) stressors such as observation or evaluation.

Regarding the impact of sensation seeking and mindfulness, the current study employed a correlational design. While this informs about natural between-individual variability, stronger effects may be expected by considering extremes or - with regard to mindfulness - employing within-subject manipulations (e.g., mindfulness training; Bühlmayer et al., 2017; Hoja et al., 2018). Still, the current study identified sensation seeking as a significant predictor of individuals' psychological response to a risk sport-specific stressor (cf. Breivik, 1999a). Moving beyond the immediate context of high risk sports, this finding bears relevance for other high risk contexts and occupations, such as firefighting, policing or the military, where individuals are confronted with similar stressors and threats to their physical integrity (Neria et al., 2000; Nieuwenhuys and Oudejans, 2010, 2011; Meland et al., 2015; Nieuwenhuys et al., 2015; Giessing et al., 2019) and analyses of sensation seeking may potentially contribute to recruitment and selection processes (e.g., Landman et al., 2016).

In the current study, only male participants were included and - hence - potential gender-specific differences in sensation seeking, mindfulness and stress responses, were not taken into account. The decision to include only male participants was deliberate and driven by the fact that females' cortisol levels can be biased by the menstrual cycle and contraceptives (Kelly et al., 2008) as well as by the fact that climbing also depends on endurance and strength and that these domains differ between the sexes. In extrapolating the current findings to the wider population, these differences should be taken into account.

Building on the current findings, future studies may include a more detailed analysis of individuals' psychological response to stress and - in context of the appraisal process (Lazarus and Folkman, 1984) - clarify if the observed stress-buffering effects of sensation seeking are caused by the primary or secondary appraisal. In addition, and in light of recent work from clinical psychology (Engel-Yeger et al., 2016; Serafini et al., 2017), analyses of sensory processing patterns could be helpful to further characterize athletes and their vulnerability to stress. Finally, in order to forward understanding of high risk sports performance, it is important to replicate the current findings and contrast 
observations with those obtained among actual risk sport athletes (e.g., Breivik, 1999a).

\section{CONCLUSION}

In conclusion, the current study showed that the personality trait sensation seeking may act as a stress "buffer" and significantly reduces individuals' psychological response (i.e., self-perceived somatic and cognitive state anxiety) to an experimentally controlled, high risk sport-specific stressor. In contrast to our hypotheses, no additional anxiety-reducing effect was observed for trait mindfulness, and neither sensation seeking nor mindfulness could explain observed variance in individuals' physiological stress response (i.e., salivary cortisol). Because of the far-reaching negative consequences of stress, identifying protective factors to secure and improve the health and performance of people who are exposed to highly demanding and risky situations (e.g., in the context of work or sports) is of critical importance. With regards to the protective influence of sensation seeking and mindfulness, the current study takes a first step in addressing this issue.

\section{DATA AVAILABILITY}

The raw data supporting the conclusions of this manuscript will be made available by the authors, without undue reservation, to any qualified researcher.

\section{ETHICS STATEMENT}

The study's design was approved by the ethical committee of the Faculty of Behavioral and Cultural Studies of Heidelberg University.

\section{AUTHOR CONTRIBUTIONS}

This manuscript at hand was mutually developed. Each author contributed to the study planning, data analysis, and

\section{REFERENCES}

Adam, E. K., and Kumari, M. (2009). Assessing salivary cortisol in large-scale, epidemiological research. Psychoneuroendocrinology 34, 1423-1436. doi: 10. 1016/j.psyneuen.2009.06.011

Allen, M. S., Greenlees, I., and Jones, M. (2013). Personality in sport: a comprehensive review. Int. Rev. Sport Exerc. Psychol. 6, 184-208. doi: 10.1016/ j.ynstr.2016.11.001

Anshel, M., and Anderson, D. (2002). Coping with acute stress in sport: linking athletes' coping style, coping strategies, affect, and motor performance. Anxiety Stress Coping 15, 193-209. doi: 10.1080/10615800290028486

Arch, J. J., and Craske, M. G. (2010). Laboratory stressors in clinically anxious and non-anxious individuals: the moderating role of mindfulness. Behav. Res. Ther. 48, 495-505. doi: 10.1016/j.brat.2010.02.005

Arijs, C., Chroni, S., Brymer, E., and Carless, D. (2017). 'Leave your ego at the door': a narrative investigation into effective wingsuit flying. Front. Psychol. 8:1985. doi: $10.3389 /$ fpsyg.2017.01985 interpretation with an additional focus on their respective area of competence. All authors contributed crucially in drafting the aim of the study, concretizing the design, and finishing the manuscript, and examined and agreed to the submitted version of the manuscript. JB, R-BH, and $\mathrm{MF}$ conducted the experiments. JB was essentially responsible for the statistical analysis with the support of CK and MS. MF interpreted the data, wrote the first draft of the manuscript together with JB, managed the communication between all authors during the development of the manuscript, assumed responsibility for being the corresponding author, and for keeping the co-authors informed of the progress through the editorial review process, the contents of the reviews, and any revisions made.

\section{FUNDING}

This study was supported by the excellence initiative "Field of Focus 4: Self-Regulation and Regulation" at the Heidelberg University (grant number: ZUK49/2 4.1.042).

\section{ACKNOWLEDGMENTS}

We gratefully acknowledge Dr. Ina Rehberger's fast laboratory analyses of the saliva samples and the contribution of her profound expertise. Special thanks to Friederike Uhlenbrock and Thomas Stoll for helping with the data collection in this study as well as to Laura Giessing and Friederike Uhlenbrock for their careful proofreading of the manuscript. We also thank the participating students. We acknowledge financial support by the Deutsche Forschungsgemeinschaft within the funding program Open Access Publishing, by the Baden-Württemberg Ministry of Science, Research and the Arts and by the Heidelberg University.

\section{SUPPLEMENTARY MATERIAL}

The Supplementary Material for this article can be found online at: https://www.frontiersin.org/articles/10.3389/fpsyg. 2019.01719/full\#supplementary-material

Beauducel, A., Strobel, A., and Brocke, B. (2003). Psychometrische eigenschaften und normen einer deutschsprachigen fassung der sensation seeking-skalen, form $\mathrm{V}$ [psychometric characteristics and norms of a german version of sensation seeking scales, form V]. Diagnostica 49, 61-72. doi: 10.1026//00121924.49.2.61

Bertrams, A., Englert, C., and Dickhäuser, O. (2010). Self-control strength in the relation between trait test anxiety and state anxiety. J. Res. Pers. 44, 738-741. doi: 10.1016/j.jrp.2010.09.005

Birrer, D., Röthlin, P., and Morgan, G. (2012). Mindfulness to enhance athletic performance: theoretical considerations and possible impact mechanisms. Mindfulness 3, 235-246. doi: 10.1007/s12671-012-0109-2

Bishop, S. R., Lau, M., Shapiro, S., Carlson, L., Anderson, N. D., and Carmody, J., et al. (2004). Mindfulness: a proposed operational definition. Clin. Psychol. Sci. Pract. 11, 230-241. doi: 10.1093/clipsy.bph077

Brand, R., Graf, K., and Ehrlenspiel, F. (2009). "Das wettkampfangst-inventar-trait” [The competitive anxiety inventory trait], in Das Wettkampfangst-Inventar Manual [The Competitive Anxiety Inventory Manual], eds R. Brand, 
F. Ehrlenspiel, and K. Graf (Bonn: Bundesinstitut für Sportwissenschaft), 15-69.

Breivik, G. (1999a). Empirical Studies of Risk Sport (Vol. B. 5). Oslo: Norges idrettshøgskole, Institutt for Samfunnsfag.

Breivik, G. (1999b). Sensation Seeking in Sport. Oslo: Norges Idrettshøgskole, Institutt for Samfunnsfag.

Breivik, G., Roth, W. T., and Jørgensen, P. E. (1998). Personality, psychological states and heart rate in novice and expert parachutists. Pers. Indiv. Dif. 25, 365-380. doi: 10.1016/S0191-8869(98)00058-0

Brown, K. W., Goodman, R. J., and Inzlicht, M. (2012a). Dispositional mindfulness and the attenuation of neural responses to emotional stimuli. Soc. Cogn. Affect. Neurosci. 8, 93-99. doi: 10.1093/scan/nss004

Brown, K. W., Weinstein, N., and Creswell, J. D. (2012b). Trait mindfulness modulates neuroendocrine and affective responses to social evaluative threat. Psychoneuroendocrinology 37, 2037-2041. doi: 10.1016/j.psyneuen.2012.04.003

Brown, K. W., and Ryan, R. M. (2003). The benefits of being present. J. Pers. Soc. Psychol. 84, 822-848. doi: 10.1037/0022-3514.84.4.822

Brown, K. W., Ryan, R. M., and Creswell, J. D. (2007). Mindfulness: theoretical foundations and evidence for its salutary effects. Psychol. Inq. 18, 211-237. doi: 10.1080/10478400701598298

Brymer, E., and Schweitzer, R. (2013). The search for freedom in extreme sports: a phenomenological exploration. Psychol. Sport Exerc. 14, 865-873. doi: 10.1016/ j.psychsport.2013.07.004

Bühlmayer, L., Birrer, D., Röthlin, P., Faude, O., and Donath, L. (2017). Effects of mindfulness practice on performance-relevant parameters and performance outcomes in sports: a meta-analytical review. Sports Med. 47, 2309-2321. doi: 10.1007/s40279-017-0752-9

Campbell, J., and Ehlert, U. (2012). Acute psychosocial stress: does the emotional stress response correspond with physiological responses? Psychoneuroendocrinology 37, 1111-1134. doi: 10.1016/j.psyneuen.2011. 12.010

Couture, S., Brown, T. G., Ouimet, M. C., Gianoulakis, C., Tremblay, J., and Carbonneau, R. (2008). Hypothalamic-pituitary-adrenal axis response to stress in male DUI recidivists. Accid. Anal. Prev. 40, 246-253. doi: 10.1016/j.aap.2007. 06.003

Creswell, J. D., and Lindsay, E. K. (2014). How does mindfulness training affect health? A mindfulness stress buffering account. Curr. Dir. Psychol. Sci. 23, 401-407. doi: 10.1177/0963721414547415

Creswell, J. D., Lindsay, E. K., Villalba, D. K., and Chin, B. (2019). Mindfulness training and physical health: mechanisms and outcomes. Psychosom. Med. 81, 224-232. doi: 10.1097/PSY.0000000000000675

Ehrlenspiel, F., Brand, R., and Graf, K. (2009). "Das wettkampfangst-inventarstate" [The competitive anxiety inventory state], in Das WettkampfangstInventar, Manual [The Competitive Anxiety Inventory, Manual], eds R. Brand, F. Ehrlenspiel, and K. Graf (Bonn: Bundesinstitut für Sportwissenschaft), 71-100.

Engel-Yeger, B., Muzio, C., Rinosi, G., Solano, P., Geoffroy, P. A., and Pompili, M., et al. (2016). Extreme sensory processing patterns and their relation with clinical conditions among individuals with major affective disorders. Psychiatry Res. 236, 112-118. doi: 10.1016/j.psychres.2015.12.022

Eysenck, M. W., Derakshan, N., Santos, R., and Calvo, M. G. (2007). Anxiety and cognitive performance: attentional control theory. Emotion 7, 336-353. doi: 10.1037/1528-3542.7.2.336

Faul, F., Erdfelder, E., Buchner, A., and Lang, A. G. (2009). Statistical power analyses using $\mathrm{G}^{*}$ Power 3.1: tests for correlation and regression analyses. Behav. Res. Methods 41, 1149-1160. doi: 10.3758/BRM.41.4.1149

Field, A. (2009). Discovering Statistics Using SPSS: (and Sex and Drugs and Rock ' $n$ ' Roll) 3rd Edn. Los Angeles, CA: Sage Publications.

Franken, R. E., Gibson, K. J., and Rowland, G. (1992). Sensation seeking and the tendency to view the world as threatening. Pers. Indiv. Dif. 13, 31-38. doi: 10.1016/0191-8869(92)90214-A

Frenkel, M. O. (2018). Der Einfluss Von Spezifischen Persönlichkeitsmerkmalen auf das Stresserleben im Sport [The Influence of Specific Personality Characteristics on the Stress Experience in Sport.], Unpublished habilitation thesis, Heidelberg University, Heidelberg.

Frenkel, M. O., Brokelmann, J., and Plessner, H. (2018a). Mental starke Kinder und Jugendliche durch Achtsamkeit? [Mentally though children and adolescents through mindfulness?]. Leistungslust 6, 28-32.
Frenkel, M. O., Heck, R. B., and Plessner, H. (2018b). Cortisol and behavioral reaction of low and high sensation seekers differ in responding to a sport-specific stressor. Anxiety Stress Coping 31, 580-593. doi: 10.1080/ 10615806.2018.1498277

Frenkel, M. O., Laborde, S., Rummel, J., Giessing, L., Kasperk, C., Plessner, H., et al. (in press). Heidelberg Risk Sport-Specific Stress Test: a paradigm to investigate the risk sport-specific psycho-physiological arousal. Front. Psychol.

Gardner, F. L., and Moore, Z. E. (2004). A Mindfulness-Acceptance-CommitmentBased Approach to Athletic Performance Enhancement: Theoretical Considerations. Orlando, FL: Elsevier Ltd.

Gardner, F. L., and Moore, Z. E. (2017). Mindfulness-based and acceptancebased interventions in sport and performance contexts. Curr. Opin. Psychol. 16, 180-184. doi: 10.1016/j.copsyc.2017.06.001

Giessing, L., Frenkel, M. O., Zinner, C., Rummel, J., Nieuwenhuys, A., Kasperk, C., et al. (2019). Effects of coping-related traits and psychophysiological stress responses on police recruits. Shooting behavior in reality-based scenarios. Front. Psychol. 10:1523. doi: 10.3389/fpsyg.2019.01523

Höfling, V., Ströhle, G., Michalak, J., and Heidenreich, T. (2011). A short version of the kentucky inventory of mindfulness skills. J. Clin. Psychol. 67, 639-645. doi: 10.1002/jclp. 20778

Hoja, S., Zirkelbach, J., and Jansen, P. (2018). Achtsamkeit - Auch ein megatrend im leistungssport? [Mindfulness - Also a megatrend in competitive sport?]. Sports Orthop. Traumatol. 34, 38-44. doi: 10.1016/j.orthtr.2017.11.001

Houge Mackenzie, S., and Brymer, E. (2018). Conceptualizing adventurous nature sport: a positive psychology perspective. Ann. Leis. Res. 13, 1-13. doi: 10.1080/ 11745398.2018.1483733

Houtman, I. L. D., and Bakker, F. C. (1989). The anxiety thermometer: a validation study. J. Pers. Assess. 53, 575-583. doi: 10.1207/s15327752jpa5 303_14

John, S., Verma, S. K., and Khanna, G. (2011). The effect of mindfulness meditation on HPA-axis in pre-competition stress in sports performance of elite shooters. Natl. J. Integr. Res. Med. 2, 15-21.

Jones, G. (1995). More than just a game: research developments and issues in competitive anxiety in sports. Br. J. Psychol. 86, 449-478. doi: 10.1111/j.20448295.1995.tb02565.x

Josefsson, T., Ivarsson, A., Gustafsson, H., Stenling, A., Lindwall, M., Tornberg R., et al. (2019). Effects of mindfulness-acceptance-commitment (MAC) on sport- specific dispositional mindfulness, emotion regulation, and self-rated athletic performance in a multiple-sport population: an RCT study. Mindfulness 10:1518. doi: 10.1007/s12671-019-01098-7

Josefsson, T., Ivarsson, A., Lindwall, M., Gustafsson, H., Stenling, A., and Böröy, J., et al. (2017). Mindfulness mechanisms in sports: mediating effects of rumination and emotion regulation on sport-specific coping. Mindfulness 8, 1354-1363. doi: 10.1007/s12671-017-0711-4

Kabat-Zinn, J. (1994). Wherever You Go, There You Are: Mindfulness Meditation in Everyday Life. New York, NJ: Hyperion.

Kabat-Zinn, J., Beall, B., and Rippe, J. (1985). A systematic mental training program based on mindfulness meditation to optimize performance in collegiate and Olympic rowers. Paper Presented at the World Congress in Sport Psychology, Copenhagen.

Kelly, M. M., Tyrka, A. R., Anderson, G. M., Price, L. H., and Carpenter, L. L. (2008). Sex differences in emotional and physiological responses to the trier social stress test. J. Behav. Ther. Exp. Psychiatry 39, 87-98. doi: 10.1016/j.jbtep. 2007.02.003

Kirschbaum, C., and Hellhammer, D. (2000). Salivary cortisol - An invaluable tool in studies of HPA function. Psychoneuroendocrinology 25:14. doi: 10.1016/ S0306-4530(00)90086-6

Kirschbaum, C., Pirke, K. M., and Hellhammer, D. H. (1993). The 'Trier Social Stress Test'. A tool for investigating psychobiological stress responses in a laboratory setting. Neuropsychobiology 28, 76-81. doi: 10.1159/000119004

Klaperski, S., von Dawans, B., Heinrichs, M., and Fuchs, R. (2013). Does the level of physical exercise affect physiological and psychological responses to psychosocial stress in women? Psychol. Sport Exerc. 14, 266-274. doi: 10.1016/j. psychsport.2012.11.003

Kudielka, B. M., Hellhammer, D. H., and Wüst, S. (2009). Why do we respond so differently? Reviewing determinants of human salivary cortisol responses to challenge. Psychoneuroendocrinology 34, 2-18. doi: 10.1016/j.psyneuen.2008. 10.004 
Landman, A., Nieuwenhuys, A., and Oudejans, R. R. D. (2016). The impact of personality traits and professional experience on police officers' shooting performance under pressure. Ergonomics 59, 950-961.

Lautenbach, F. (2017). A laboratory study on attentional bias as an underlying mechanism affecting the link between cortisol and performance, leading to a discussion on the nature of the stressor (artificial vs. psychosocial). Physiol. Behav. 175, 9-15. doi: 10.1016/j.physbeh.2017.03.022

Lautenbach, F., Laborde, S., Achtzehn, S., and Raab, M. (2014). Preliminary evidence of salivary cortisol predicting performance in a controlled setting. Psychoneuroendocrinology 42, 218-224. doi: 10.1016/j.psyneuen.2014.01.011

Lazarus, R. S. (2000). How emotions influence performance in competitive sports. Sport Psychol. 14, 229-252. doi: 10.1123/tsp.14.3.229

Lazarus, R. S., and Folkman, S. (1984). Stress, Appraisal, and Coping. New York, NJ: Springer Publishing Company.

Manigault, A. W., Woody, A., Zoccola, P. M., and Dickerson, S. S. (2018). Trait mindfulness predicts the presence but not the magnitude of cortisol responses to acute stress. Psychoneuroendocrinology 90, 29-34. doi: 10.1016/j.psyneuen. 2018.01.022

Meland, A., Ishimatsu, K., Pensgaard, A. M., Wagstaff, A., Fonne, V., and Garde, A. H., et al. (2015). Impact of mindfulness training on physiological measures of stress and objective measures of attention control in a military helicopter unit. Int. J. Aviat. Psychol. 25, 191-208. doi: 10.1080/10508414.2015.1162639

Miller, R., and Plessow, F. (2013). Transformation techniques for cross-sectional and longitudinal endocrine data: application to salivary cortisol concentrations. Psychoneuroendocrinology 38, 941-946. doi: 10.1016/j.psyneuen.2012. 09.013

Neria, Y., Solomon, Z., Ginzburg, K., and Dekel, R. (2000). Sensation seeking, wartime performance, and long-term adjustment among Israeli war veterans. Pers. Indiv. Dif. 29, 921-932. doi: 10.1016/S0191-8869(99)00243-3

Nieuwenhuys, A., and Oudejans, R. R. D. (2010). Effects of anxiety on handgun shooting behavior of police officers: a pilot study. Anxiety Stress Coping 23, 225-233. doi: 10.1080/10615800902977494

Nieuwenhuys, A., and Oudejans, R. R. D. (2011). Training with anxiety: short- and long-term effects on police officers' shooting behavior under pressure. Cogn. Process. 12, 277-288. doi: 10.1007/s10339-011-0396-x

Nieuwenhuys, A., and Oudejans, R. R. D. (2012). Anxiety and perceptualmotor performance: toward an integrated model of concepts, mechanisms, and processes. Psychol. Res. 76, 747-759. doi: 10.1007/s00426-0110384- $\mathrm{x}$

Nieuwenhuys, A., and Oudejans, R. R. D. (2017). Anxiety and performance: perceptual-motor behavior in high-pressure contexts. Curr. Opin. Psychol. 16, 28-33. doi: 10.1016/j.copsyc.2017.03.019

Nieuwenhuys, A., Pijpers, J. R., Oudejans, R. R. D., and Bakker, F. C. (2008). The influence of anxiety on visual attention in climbing. J. Sport Exerc. Psychol. 30, 171-185. doi: 10.1123/jsep.30.2.171

Nieuwenhuys, A., Savelsbergh, G. J. P., and Oudejans, R. R. D. (2015). Persistence of threat-induced erros in police officers' shooting decisions. Appl. Ergon. 48, 263-272. doi: 10.1016/j.apergo.2014.12.006

Paulus, M. P., Potterat, E. G., Taylor, M. K., Van Orden, K. F., Bauman, J., and Momen, N., et al. (2009). A neuroscience approach to optimizing brain resources for human performance in extreme environments. Neurosci. Biobehav. Rev. 33, 1080-1088. doi: 10.1016/j.neubiorev.2009.05.003

Plessner, H., Unkelbach, C., Memmert, D., Baltes, A., and Kolb, A. (2009). Regulatory fit as a determinant of sport performance: how to succeed in a soccer penaltyshooting. Psychol. Sport Exerc. 10, 108-115. doi: 10.1016/j.psychsport. 2008.02.001

Pruessner, J. C., Kirschbaum, C., Meinlschmid, G., and Hellhammer, D. H. (2003). Two formulas for computation of the area under the curve represent measures of total hormone concentration versus time-dependent change. Psychoneuroendocrinology 28, 916-931. doi: 10.1016/S0306-4530(02) 00108-7

Röthlin, P., Horvath, S., Birrer, D., and Grosse Holtforth, M. (2016). Mindfulness promotes the ability to deliver performance in highly demanding situations. Mindfulness 7, 727-733. doi: 10.1007/s12671-016-0512-1

Ruedl, G., Abart, M., Ledochowski, L., Burtscher, M., and Kopp, M. (2012). Selfreported risk taking and risk compensation in skiers and snowboarders are associated with sensation seeking. Accid. Anal. Prev. 48, 292-296. doi: 10.1016/ j.aap.2012.01.031

Schultheiss, O. C., and Stanton, S. J. (2009). “Assessment of salivary hormones”, in Methods in Social Neuroscience, eds E. Harmon-Jones, and J. S. Beer (New York, NJ: Guilford Press), 17-44.

Serafini, G., Gonda, X., Canepa, G., Pompili, M., Rihmer, Z., Amore, M., et al. (2017). Extreme sensory processing patterns show a complex association with depression, and impulsivity, alexithymia, and hopelessness. J. Affect. Disord. 210, 249-257. doi: 10.1016/j.jad.2016.12.019

Shabani, S., Dehghani, M., Hedayati, M., and Rezaei, O. (2011). Relationship of serum serotonin and salivary cortisol with sensation seeking. Int. J. Psychophysiol. 81, 225-229. doi: 10.1016/j.ijpsycho.2011. 06.015

Shapiro, S. L., Carlson, L. E., Astin, J. A., and Freedman, B. (2006). Mechanisms of mindfulness. J. Clin. Psychol. 62, 373-386. doi: 10.1002/jclp.20237

Smith, R., Ptacek, J., and Smoll, F. (1992). Sensation seeking, stress, and adolescent injuries: a test of stress-buffering, risk-taking, and coping skills hypotheses. J. Pers. Soc. Psychol. 62, 1016-1024. doi: 10.1037/00223514.62.6.1016

Sothmann, M. S., Buckworth, J., Claytor, R. P., Cox, R. H., White-Welkley, J. E., and Dishman, R. K. (1996). Exercise training and the cross-stressor adaptation hypothesis. Exerc. Sport Sci. Rev. 24, 267-288.

Tabachnick, B. G., and Fidell, L. S. (2007). Using Multivariate Statistics. Boston, MA: Pearson.

Wirtz, M. (2004). Über das problem fehlender Werte: wie der Einfluss fehlender informationen auf analyseergebnisse entdeckt und reduziert werden kann [about the problem of missing values: how to detect and reduce the influence of missing information on analyses results]. Die Rehabilitation 43, 109-115. doi: 10.1055/s-2003-814839

Zschucke, E., Renneberg, B., Dimeo, F., Wüstenberg, T., and Ströhle, A. (2015). The stress-buffering effect of acute exercise: evidence for HPA axis negative feedback. Psychoneuroendocrinology 51, 414-425. doi: 10.1016/j.psyneuen.2014. 10.019

Zuckerman, M. (1994). Behavioral Expressions and Biosocial Bases of Sensation Seeking. Cambridge: Cambridge University Press.

Zuckerman, M. (1996). The psychobiological model for impulsive unsocialized sensation seeking: a comparative approach. Neuropsychobiology 34, 125-129. doi: $10.1159 / 000119303$

Zuckerman, M. (2007). Sensation Seeking and Risky Behavior. Washington: American Psychological Association.

Conflict of Interest Statement: The authors declare that the research was conducted in the absence of any commercial or financial relationships that could be construed as a potential conflict of interest.

Copyright (c) 2019 Frenkel, Brokelmann, Nieuwenhuys, Heck, Kasperk, Stoffel and Plessner. This is an open-access article distributed under the terms of the Creative Commons Attribution License (CC BY). The use, distribution or reproduction in other forums is permitted, provided the original author(s) and the copyright owner(s) are credited and that the original publication in this journal is cited, in accordance with accepted academic practice. No use, distribution or reproduction is permitted which does not comply with these terms. 